\title{
Dense Low-Coordination Phases of Lithium
}

\author{
Chris J. Pickard \\ Scottish Universities Physics Alliance, School of Physics and Astronomy, University of St. Andrews, \\ St Andrews, KY16 9SS, United Kingdom \\ R. J. Needs \\ Theory of Condensed Matter Group, Cavendish Laboratory, Cambridge CB3 OHE, United Kingdom
} (Received 17 September 2008; published 7 April 2009)

\begin{abstract}
$A b$ initio density-functional-theory calculations and a structure-searching technique are used to identify candidate high-pressure phases of lithium $(\mathrm{Li})$. We predict threefold coordinated structures to be stable in the pressure range 40-450 GPa and fourfold structures at higher pressures. We describe these lowcoordination phases as elemental electrides. All of the stable phases are metallic but the Cmca-24 structure, and two distortions of it which are marginally the most stable in the pressure range 86-106 GPa, are nearly semiconducting with densities of electronic states at the Fermi energy of only a few percent of the free-electron value.
\end{abstract}

DOI: 10.1103/PhysRevLett.102.146401

PACS numbers: 71.15.Nc, 61.66. $-\mathrm{f}, 62.50 .-\mathrm{p}$

In the close-packed structures adopted by $\mathrm{Li}$ at low pressures [1] the $2 s$ electrons combine to form a half-filled nearly-free-electron band, consistent with the picture of a simple alkali metal. However, strong deviations from the canonical picture develop at higher pressures. Overlap of the Li $1 s$ core electrons on neighboring atoms under strong compression has a profound effect upon the structures, which adopt lower coordination structures [2,3].

The close-packed face-centered-cubic (fcc) phase of Li transforms to a structure of $I \overline{4} 3 d$ space group symmetry [4] at $\sim 40 \mathrm{GPa}$, via an intermediate phase of $R \overline{3} m$ symmetry [3]. The $R \overline{3} m$ phase has a distorted fcc structure, but the $I \overline{4} 3 d$ structure departs very substantially from close packing. $I \overline{4} 3 d$ has three short interatomic distances giving threefold coordination. Calculations show that the valence electronic density of states (e-DOS) dips around the Fermi energy $\left(E_{F}\right)$ [3]. These features are consistent with the picture that reduced coordination numbers at high pressures arise from a Jahn-Teller-like distortion [2]. Calculations of the high-pressure phases also show an accumulation of electronic charge density within the interstitial region as the $2 s$ electron density is pushed away from the atomic cores by Coulomb repulsion, Pauli exclusion, and the orthogonality of core and valence orbitals $[2,3,5]$.

Experiments suggest another phase transition at 60 $70 \mathrm{GPa}$, although the new structure has not been determined [6,7]. A number of theoretical studies have sought to identify candidate phases at higher pressures $[2,8,9]$. A DFT study by Rousseau et al. [8] found a structure of Cmca symmetry to be more stable than $I \overline{4} 3 d$ above $88 \mathrm{GPa}$. This structure is named $\mathrm{Cmca}-24$, appending the number of atoms in the conventional unit cell [8]. Cmca-24 consists of threefold coordinated atoms [8].

Ma et al. [9] studied high-pressure phases of Li using a combination of $a b$ initio DFT methods and an evolutionary search algorithm, finding a new structure of $P 4_{1} 32$ symmetry which they calculated to be more stable than Cmca-24 above $300 \mathrm{GPa}$. This structure contains sixfold coordinated atoms. On the other hand, recent hightemperature DFT molecular dynamics simulations found fourfold coordinated $\mathrm{Li}$ atoms in the pressure range 150$810 \mathrm{GPa}[10]$.

Superconductivity has been observed in $\mathrm{Li}$ up to $\sim 85 \mathrm{GPa}$, indicating metallic behavior $[6,11,12]$. However, calculations have suggested that semimetallic or even semiconducting behavior might occur at high pressures [2]. Increases in the electrical resistance of highly compressed Li have also been observed in shockwave experiments, lending support to this suggestion $[13,14]$.

The lack of experimental information on the highpressure phases of Li beyond $I \overline{4} 3 d$, the possibility of semiconducting behavior at high pressures, and differences between the various theoretical predictions motivates the present study. We have searched for candidate highpressure phases of $\mathrm{Li}$ using a combination of $a b$ initio calculations and "random structure searching" which we refer to as "AIRSS". This approach has been applied successfully to a number of systems [15-18]. An ensemble of structures is chosen by first generating random unit cell translation vectors and renormalizing the resulting cell volumes to lie within some reasonable range. The atoms are then placed at random positions and the cell shapes and atomic positions are relaxed at a fixed pressure to a minimum in the enthalpy.

We performed searches at $50 \mathrm{GPa}$ (with 8, 12, and 16 atoms per cell), $75 \mathrm{GPa}(12,16,24$ atoms), $100 \mathrm{GPa}(8,12$, $14,16,18,24$ atoms) $200 \mathrm{GPa}(8,12,16$ atoms), $400 \mathrm{GPa}$ $(4,8,12,16$ atoms), $600 \mathrm{GPa}(8,12,16$ atoms), and searches at 1000 and $2000 \mathrm{GPa}$ with 8-atom cells. We 
also performed a search at $100 \mathrm{GPa}$ with 24 atoms whose positions were constrained to conform to the $P 2_{1}$ space group which contains two symmetry operations. We used the CASTEP plane-wave code [19], ultrasoft pseudopotentials [20], and the Perdew-Burke-Ernzerhof (PBE) generalized gradient approximation (GGA) density functional [21]. For the searches we used a plane-wave basis set cutoff of $350 \mathrm{eV}$ and the Brillouin zone integrations were performed using a $k$-point grid of spacing $2 \pi \times 0.05 \AA^{-1}$. The most interesting structures were further relaxed at a higher level of accuracy consisting of a basis set cutoff of $420 \mathrm{eV}$ and a $k$-point grid spacing of $2 \pi \times 0.03 \AA^{-1}$. Very high quality pseudopotentials in which all three electrons are treated explicitly must be used to obtain accurate results. A set of test calculations were performed using pseudopotentials with progressively smaller core radii and the bare Coulomb potential. We concluded that the errors in the enthalpy differences between phases arising from a pseudopotential of core radius 1.2 a.u. are smaller than $10 \mathrm{meV}$ per atom. This pseudopotential was used to obtain the results reported here.

The enthalpies of the more stable phases, calculated at the higher level of accuracy, are plotted as a function of pressure in Fig. 1, and the details of the structures are reported in the auxiliary material [22]. We find the experimentally observed $I \overline{4} 3 d$ phase to be stable in a range of pressures up to $\sim 86 \mathrm{GPa}$, at which point it becomes unstable to a quite different structure of $\mathrm{Pbca}$ symmetry. The $\mathrm{Pbca}$ structure is a distortion of Cmca-24 (which has a 12-atom primitive cell) into a 24-atom primitive cell, as illustrated in Fig. 2. At $\sim 98 \mathrm{GPa}$ a distortion of $\mathrm{Cmca}-24$ with $A b a 2$ symmetry and a 12 -atom primitive cell be-

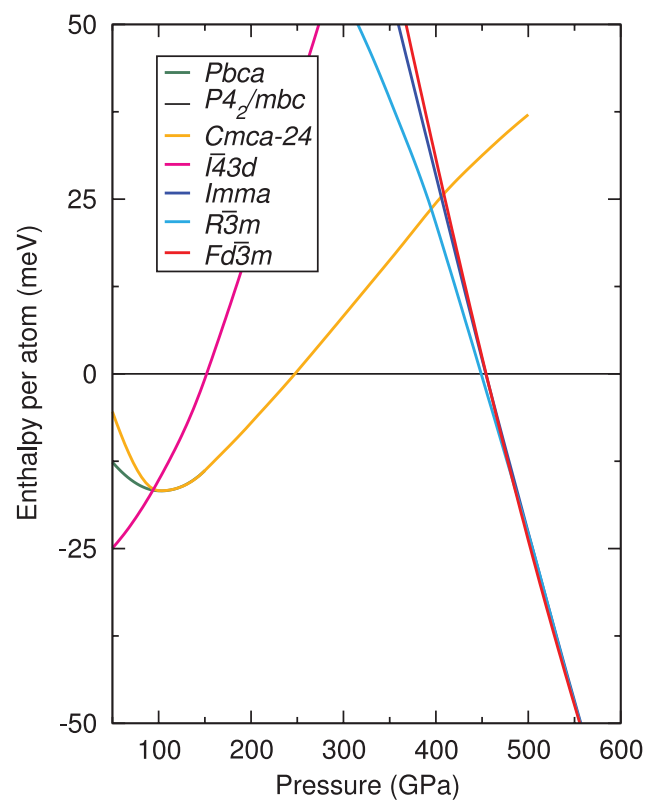

FIG. 1 (color). Variation of the enthalpies of phases of Li with pressure. Differences in enthalpy from the $P 4_{2} / m b c$ phase are plotted. comes slightly more stable than Pbca. (The enthalpy of $A b a 2$ is not plotted in Fig. 1 as it is indistinguishable from Cmca-24 on this scale.) The enthalpies of the Pbca, Aba2, and $\mathrm{Cmca}-24$ phases differ by less than $1 \mathrm{meV}$ per atom at pressures above $\sim 85 \mathrm{GPa}$, which is smaller than our calculational accuracy, but it is clear from Fig. 1 that the $\mathrm{Pbca}$ phase is favored at lower pressures and that the structures tend to the higher-symmetry $\mathrm{Cmca}-24$ phase at higher pressures.

All of the low-enthalpy high-pressure phases of Li show a substantial accumulation of electronic charge density within the interstitial regions, as was found in previous theoretical studies $[2,3,8]$. The valence e-DOS of the $I \overline{4} 3 d$, Cmca-24, and the much higher enthalpy fcc phase are shown in Fig. 3(a) at $75 \mathrm{GPa}$. (The occupied e-DOS of the $\mathrm{Pbca}, \mathrm{Aba}$, and $\mathrm{Cmca}-24$ phases are very similar, see the auxiliary material [22].) The occupied valence bandwidths are 35\%-40\% smaller than the free-electron bandwidths at the same densities. This narrowing arises because the valence electrons are pushed away from the cores and lie within the interstitial regions. Neighboring interstitial regions are partially cutoff from one another by the cores and the occupied valence bandwidth is reduced [5]. The band narrowing increases the e-DOS in the lower portion of the valence band, and the e-DOS at $E_{F}$ of the fcc phase is $210 \%$ of the free-electron value at the same density. A structure with a large e-DOS at $E_{F}$ is susceptible to a JahnTeller-like distortion and the more stable $I \overline{4} 3 d$, Pbca, $A b a 2$, and $C m c a-24$ phases show very pronounced dips in their e-DOS around $E_{F}$. The e-DOS at $E_{F}$ of $I \overline{4} 3 d$ is $72 \%$ of the free-electron value. The occupied valence bandwidths of $I \overline{4} 3 \mathrm{~d}$ and $\mathrm{Cmca}-24$ actually decrease as the pressure is increased from 75 to $100 \mathrm{GPa}$, while at the same time the e-DOS at $E_{F}$ decreases. The e-DOS at $E_{F}$ of $C m c a-24$ is only $5 \%$ of the free-electron value at $75 \mathrm{GPa}$, and it falls by a factor of 2 by $100 \mathrm{GPa}$.

The AIRSS method yields structures which are stable against zone-center phonon distortions (including elastic distortions) within the unit cell used for the searches. Additional calculations indicated that the Pbca, Aba2, and $\mathrm{Cmca}-24$ phases have some soft phonon frequencies at pressures around $100 \mathrm{GPa}$ which are very sensitive to the quality of the Brillouin zone integrations. It is possible that

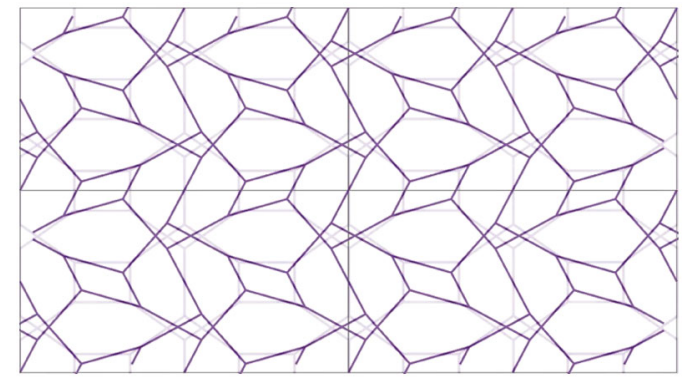

FIG. 2 (color online). Frameworks of the Pbca (dark) and Cmca-24 (light) structures at $75 \mathrm{GPa}$. The cell vectors of Pbca have been scaled slightly to equal those of Cmca-24. 

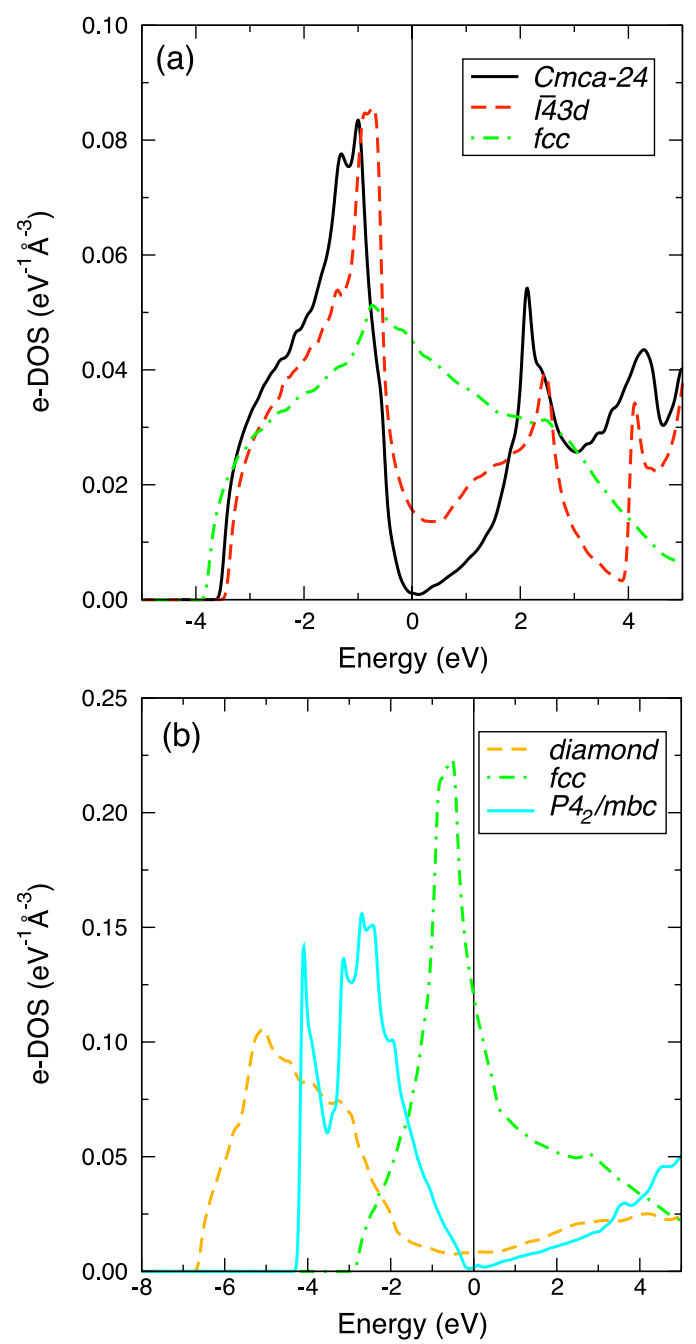

FIG. 3 (color online). Valence electronic densities of states (eDOS) of selected phases (a) at $75 \mathrm{GPa}$ and (b) at $450 \mathrm{GPa}$. The zero of energy is placed at $E_{F}$.

other structures with similar bonding might exist in this pressure range which are slightly more stable than the ones we have found. The existence of several similar structures which are almost degenerate in enthalpy suggests that disordered structures might be obtained in experiments. The combination of structural disorder and very low eDOS around $E_{F}$ could even result in semiconducting behavior.

We find the Cmca-24 phase to be the most stable above $106 \mathrm{GPa}$, and Tse et al. [23] have shown that this phase has stable phonon modes at high pressures. We have found several new structures at higher pressures. A structure of $\mathrm{P}_{2} / \mathrm{mbc}$ symmetry is shown in Fig. 4 which becomes more stable than Cmca-24 at $247 \mathrm{GPa}$. We found the phonons of $P 4_{2} / m b c$ to be stable across the entire Brillouin zone, see the auxiliary material [22]. $P 4_{2} / m b c$ is predicted to be stable up to $449 \mathrm{GPa}$ where it yields to a new structure of $R \overline{3} \mathrm{~m}$ symmetry, which is in turn replaced by the diamond structure (space group $F d \overline{3} m$ ) at $483 \mathrm{GPa}$. The $R \overline{3} \mathrm{~m}$, diamond structure, and Imma phases are almost

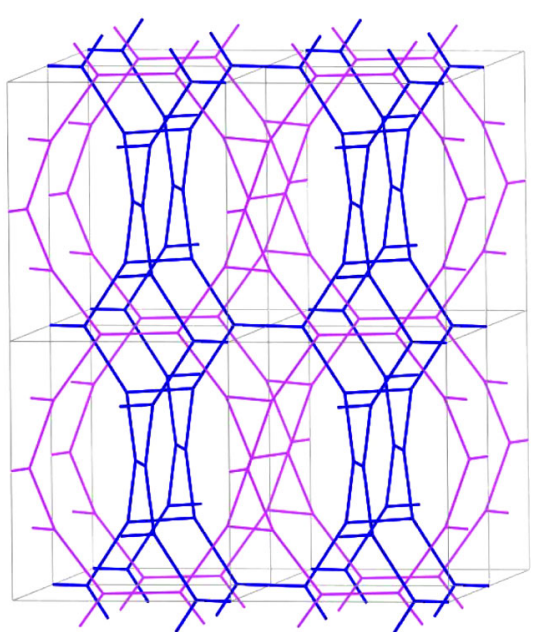

FIG. 4 (color online). Framework of the $P 4_{2} / m b c$ structure at $250 \mathrm{GPa}$. The different colors indicate the two interpenetrating threefold coordinated networks.

degenerate above $\sim 450 \mathrm{GPa}$ and, in fact, the $R \overline{3} \mathrm{~m}$ and Imma structures are small distortions of the diamond structure at these pressures, which explains why they are so nearly degenerate. The diamond structure is calculated to be stable to pressures beyond $1 \mathrm{TPa}$.

$\mathrm{P}_{2} / \mathrm{mbc}$ is a threefold coordinated structure composed of two separate but inter-penetrating threefold coordinated networks, as illustrated in Fig. 4. The $R \overline{3} m$, diamond, and Imma structures have fourfold coordination. The diamond structure is well known as occurring in carbon and also in silicon and germanium, while the Imma structure occurs in silicon and germanium [24]. We calculated the $P 4_{1} 32$ sixfold coordinated structure proposed by Ma et al. [9] to be roughly $100 \mathrm{meV}$ per atom higher in enthalpy than our $P 4_{2} / m b c$ structure over the range $200-600 \mathrm{GPa}$, and it is never the most stable structure. The reason for this discrepancy is not entirely clear to us although, as noted above, the results are sensitive to the pseudopotential core radius, and we have obtained similar results to Ma et al. when using radii which are too large. We therefore conclude that the coordination number of $\mathrm{Li}$ atoms increases from three to four at high pressures, in agreement with Tamblyn et al. [10] Our estimate of the transition pressure of $449 \mathrm{GPa}$ is likely to be an overestimate because we have neglected quantum zero point motion which favors more closely packed structures. It is expected that all elements will form close-packed structures under sufficient compression, but this must occur at extremely high pressures in $\mathrm{Li}$.

The e-DOS of the higher-pressure $P 4_{2} / m b c$ and diamond-structure phases are shown in Fig. 3(b) at $450 \mathrm{GPa}$, which is close to their coexistence pressure, along with the e-DOS of the fcc phase. At this pressure the occupied valence bandwidths are $19 \%$ (fcc), $24 \%$ $\left(\mathrm{P}_{2} / \mathrm{mbc}\right.$ ), and $38 \%$ (diamond) of their respective freeelectron values. The e-DOS of fcc has a strong peak just below $E_{F}$ and the e-DOS at $E_{F}$ is $44 \%$ of the free-electron 
value. The e-DOS of the diamond structure and $P 4_{2} / m b c$ phases show pronounced dips around $E_{F}$. The e-DOS of the diamond structure at $E_{F}$ is $29 \%$ of the free-electron value, while that of $P 4_{2} / \mathrm{mbc}$ is only $6 \%$ of the freeelectron value. The mechanisms of band narrowing in the lower portion of the valence band and the increased stability of phases such as $P 4_{2} / m b c$ and the diamond structure associated with a reduction in the e-DOS near $E_{F}$ still operate at very high pressures.

The bands arising from the $1 s$ core electrons are $\sim 45 \mathrm{eV}$ below $E_{F}$ at $75 \mathrm{GPa}$ and $\sim 50 \mathrm{eV}$ below at $450 \mathrm{GPa}$. The structures with short nearest-neighbor distances tend to have wider core bandwidths, and the core bandwidths increase with pressure. At $75 \mathrm{GPa}$, the core bandwidths are substantially smaller than the occupied valence bandwidths although in $\mathrm{Cmca-24}$, which has the shortest nearest-neighbor separation of the structures appearing in Fig. 3(a), the core bandwidth is $50 \%$ of the occupied valence bandwidth. At $450 \mathrm{GPa}$ the core bandwidths are considerably larger, and for the structures appearing in Fig. 3(b) the core bandwidth is roughly twice the occupied valence bandwidth.

Lithium develops a novel electronic structure under high pressures. The valence electrons are pushed away from the ionic cores and form piles of charge at interstitial locations. These piles of electronic charge are rather isolated from one another, and the occupied valence bandwidths of the high-pressure phases are narrower than the corresponding free-electron values, in accord with the picture developed by Rousseau and Ashcroft [5]. The situation is strongly reminiscent of an "electride" [25], in which the electrons play the role of the anions. At high pressures we predict the most stable structure to consist of $\mathrm{Li}$ ions arranged in the diamond structure. In this structure the $\mathrm{Li}$ ions and the valence electron sites form interpenetrating diamond lattices and, taking the Li and electron sites together, they form the B32 (NaTl-type) Zintl structure. This structure is adopted by several ionic $A B$ compounds with $A^{+}$ions from group I of the periodic table $(\mathrm{Li}, \mathrm{Na}$, and $\mathrm{K})$ and $B^{-}$ions from group III (Al, Ga, In, and Tl) [26].

In conclusion, we find that the most stable phases of $\mathrm{Li}$ have threefold coordination in the pressure range 40$450 \mathrm{GPa}$, but fourfold coordination from $450 \mathrm{GPa}$ up to pressures beyond $1 \mathrm{TPa}$. The return to close-packed structures must occur at extremely high pressures beyond the reach of current static diamond-anvil-cell experiments. We found new distortions of the $\mathrm{Cmca}-24$ structure, including one of $\mathrm{Pbca}$ symmetry which is energetically favorable at lower pressures. Our prediction of several nearly degenerate phases in the pressure range 86-110 GPa suggests the formation of disordered structures which, when combined with the very low e-DOS at $E_{F}$ might result in semiconducting behavior. We predict that the $\mathrm{Cmca}-24$ structure becomes unstable to a $P 4_{2} / m b c$ phase at $247 \mathrm{GPa}$, and that at still higher pressures a diamond structure is formed. We have suggested that the high-pressure phases of Li can be described as elemental electrides.

The authors were supported by the Engineering and Physical Sciences Research Council (EPSRC) of the UK.

[1] C. S. Barrett, Acta Crystallogr. 9, 671 (1956).

[2] J.B. Neaton and N.W. Ashcroft, Nature (London) 400, 141 (1999).

[3] M. Hanfland, K. Syassen, N.E. Christensen, and D. L. Novikov, Nature (London) 408, 174 (2000).

[4] We refer to the symmetries of structures by their short Hermann-Mauguin space group symbols.

[5] B. Rousseau and N. W. Ashcroft, Phys. Rev. Lett. 101, 046407 (2008).

[6] V. V. Struzhkin, M. I. Eremets, W. Gan, H.-k. Mao, and R. J. Hemley, Science 298, 1213 (2002).

[7] A.F. Goncharov, V. V. Struzhkin, H.-k. Mao, and R. J. Hemley, Phys. Rev. B 71, 184114 (2005).

[8] R. Rousseau, K. Uehara, D. D. Klug, and J. S. Tse, Chem. Phys. Chem. 6, 1703 (2005).

[9] Y. Ma, A. R. Oganov, and Y. Xie, Phys. Rev. B 78, 014102 (2008).

[10] I. Tamblyn, J.-Y. Raty, and S. A. Bonev, Phys. Rev. Lett. 101, 075703 (2008).

[11] K. Shimizu, H. Ishikawa, D. Takao, T. Yagi, and K. Amaya, Nature (London) 419, 597 (2002).

[12] K. Shimizu, D. Takao, S. Furomoto, and K. Amaya, Physica (Amsterdam) 408C, 750 (2004).

[13] V.E. Fortov, V. V. Yakushev, K.L. Kagan, I. V. Lomonosov, V.I. Postnov, T. I. Yakusheva, and A. N. Kuryanchik, JETP Lett. 74, 418 (2001).

[14] M. Bastea and S. Bastea, Phys. Rev. B 65, 193104 (2002).

[15] C. J. Pickard and R. J. Needs, Phys. Rev. Lett. 97, 045504 (2006).

[16] C. J. Pickard and R. J. Needs, Nature Phys. 3, 473 (2007).

[17] C. J. Pickard and R. J. Needs, Phys. Rev. B 76, 144114 (2007).

[18] C. J. Pickard and R. J. Needs, Nature Mater. 7, 775 (2008).

[19] S. J. Clark, M. D. Segall, C. J. Pickard, P. J. Hasnip, M. I. J. Probert, K. Refson, and M. C. Payne, Z. Kristallogr. 220, 567 (2005).

[20] D. Vanderbilt, Phys. Rev. B 41, 7892 (1990).

[21] J. P. Perdew, K. Burke, and M. Ernzerhof, Phys. Rev. Lett. 77, 3865 (1996).

[22] See EPAPS Document No. E-PRLTAO-102-074916 for details of new structures, phonons of $P 4_{2} / m b c$, and

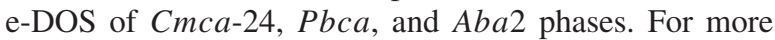
information on EPAPS, see http://www.aip.org/pubservs/ epaps.html.

[23] J.S. Tse, D. D. Klug, and T. Iitaka, Phys. Rev. B 73, 212301 (2006).

[24] A. Mujica, A. Rubio, A. Muñoz, and R. J. Needs, Rev. Mod. Phys. 75, 863 (2003).

[25] J. L. Dye, Science 301, 607 (2003).

[26] J. Evers and G. Oehlinger, Inorg. Chem. 39, 628 (2000). 